\title{
Differential orientations of the DNA-binding domain and carboxy- terminal dimerization interface regulate binding site selection by nuclear receptor heterodimers
}

\author{
Riki Kurokawa, ${ }^{1}$ Victor C. Yu, ${ }^{2}$ Anders Näär, ${ }^{3,4}$ Seiko Kyakumoto, ${ }^{1,6}$ Zhihua Han, \\ Stephanie Silverman, ${ }^{1}$ Michael G. Rosenfeld, ${ }^{2,3}$ and Christopher K. Glass, ${ }^{1,3,5}$ \\ ${ }^{1}$ Divisions of Cellular and Molecular Medicine and Endocrinology and Metabolism, ${ }^{2}$ Howard Hughes Medical Institute, \\ ${ }^{3}$ Center for Molecular Genetics, and ${ }^{4}$ Group in Molecular Pathology School of Medicine, University of California, San Diego, \\ La Jolla, California 92093-0656 USA.
}

Retinoic acid, thyroid hormone, and vitamin D receptors preferentially activate target genes through response elements that consist of direct repeat arrangements of a core recognition motif of consensus sequence AGGTCA. We present evidence that the preference for direct repeat elements arises from two fundamental differences from steroid hormone receptors. First, retinoic acid, thyroid hormone, and vitamin $D$ receptors are demonstrated to preferentially form heterodimers with the retinoid $\mathrm{X}$ receptors. These interactions are mediated by the carboxy-terminal dimerization interface, with heterodimer preference specified by actions of the DNA-binding domain. Second, the DNA-binding domains of heterodimeric receptors appear to be rotationally flexible with respect to the carboxy-terminal dimerization interface. Several independent lines of evidence suggest that, relative to the retinoid $X$ and steroid hormone receptors, the DNA-binding domain of the thyroid hormone receptor is preferentially rotated by $\sim 180^{\circ}$ with respect to its carboxy-terminal dimerization interface. As a result, solution interactions between the carboxy-terminal dimerization interfaces of the retinoid $X$ and thyroid hormone receptors are predicted to lead to the preferential alignment of their respective DNA-binding domains in a direct repeat configuration. This alignment would position the retinoid $X$ receptor over the upstream recognition motif of direct repeat response elements. Differential orientations of the DNA-binding domain, which contribute to the polarity of heterodimer binding, are regulated by a short sequence (the $A$ box) that is located between the conserved DNA-binding and carboxy-terminal dimerization domains.

[Key Words: Nuclear receptor heterodimers; DNA-binding domain; carboxy-terminal dimerization interface] Received April 9, 1993; revised version accepted May 13, 1993.

The thyroid hormone, retinoic acid, and vitamin $\mathrm{D}$ receptors (TR, RAR, and VDR, respectively) are members of the nuclear receptor superfamily of ligand-dependent transcription factors that interact with response elements in target genes and thereby control diverse aspects of development and homeostasis (Evans 1988; Beato 1989; Glass and Rosenfeld 1991). A central problem in understanding the actions of these and related nuclear receptors is the elucidation of the molecular mechanisms by which target genes are recognized. Studies of the DNA-binding properties of the TR, RAR, and VDR

\footnotetext{
${ }^{5}$ Corresponding author.

'Present address: Department of Biochemistry, Iwate Medical University, School of Dentistry, Iwate 020, Japan.
}

have revealed two fundamental differences from steroid hormone receptors with respect to the mechanisms by which they interact with DNA. First, in contrast to the estrogen and glucocorticoid receptors, which appear to bind to DNA exclusively as homodimers, the TRs or RARs preferentially interact with their cognate response elements as components of heterodimeric complexes containing retinoid X receptors (RXRs) (Yu et al. 1991; Kliewer et al. 1992; Leid et al. 1992; Marks et al. 1992; Zhang et al. 1992) and perhaps other nuclear proteins (Murray and Towle 1989; Burnside et al. 1990; Glass et al. 1990; Liao et al. 1990). Second, whereas steroid hormone receptors preferentially bind to response elements consisting of a palindromic arrangement of a conserved recognition motif, heterodimers of the TR or RAR complexed with the RXR bind with high affinity and activate 
transcription from response elements consisting of direct repeat, palindromic, and inverted palindromic arrangements of a core recognition motif of the consensus sequence AGGTCA /Glass et al. 1988; Umesono et al. 1988, 1991; Baniahmad et al. 1990; Näär et al. 1991; Carlberg et al. 1993; Tini et al. 1993).

Recognition of specific base pairs within the corebinding motif is mediated by the highly conserved DNAbinding domain that defines the nuclear receptor superfamily. This domain contains two interdependent zinc finger structures that form major groove contacts with base pairs that define the core recognition motif (Luisi et al. 1991 and references therein). Studies of the DNAbinding domains of the RXR and the orphan receptor NGFIb have indicated that amino acids immediately carboxy-terminal to the zinc finger structures also participate in DNA recognition (Wilson et al. 1992). A cluster of amino acids, termed the $\mathrm{T}$ box, was identified adjacent to the RXR zinc finger structures, which was predicted to be involved in defining part of the dimerization interface between RXR homodimers bound to an RXR-specific response element. An adjacent cluster of amino acids, termed the A box, was identified in NGFIb, which was necessary for recognition of specific base pairs at the $5^{\prime}$ end of the core recognition motif. Although the region corresponding to the $\mathrm{A}$ and $\mathrm{T}$ boxes is not conserved between different subclasses of nuclear receptors (e.g., RXR vs. RAR vs. TR), it is highly conserved within a subclass (e.g., $R X R \alpha$ vs. $\operatorname{RXR} \beta$ vs. $R X R \gamma$ ). In addition to the DNA-binding domain, high-affinity binding of nuclear receptor dimers or heterodimers is dependent on a carboxy-terminal dimerization function present within the ligand-binding domains of each receptor (Tsai et al. 1988; Glass et al. 1989; Fawell et al. 1990; Yu et al. 1991; Leid et al. 1992).

The relative orientation and spacing of the core recognition motifs play essential roles in the specificity of DNA-binding and transcriptional activation. A palindromic arrangement (AGGTCA-TGACCT, TRE-pal), functions as a common response element for TR /Glass et al. 1988), RAR (Umesono et al. 1988), and RXR (Mangelsdorf et al. 1990). In contrast, when arranged as direct repeats, the presence of 3,4 , or 2 and 5 bp between the core motifs dictates preferential binding and transcriptional activation responses by VDRs, TRs, and RARs, respectively (Näär et al. 1991; Rottman et al. 1991; Umesono et al. 1991; Durand et al. 1992).

In this paper we have utilized a binding site selection strategy to demonstrate that heterodimers between the RXR and RAR, TR or VDR preferentially select direct repeat arrangements of the core-binding motif. We propose that direct repeat-binding sites are preferentially recognized by heterodimers because, as compared with the RXR, the DNA-binding domains of the TR, VDR, and RAR are rotated by $\sim 180^{\circ}$ with respect to the carboxy-terminal dimerization interface. Solution interactions between the carboxy-terminal interfaces of the RXR and its heterodimeric partners therefore result in the alignment of their respective DNA-binding domains in a direct repeat configuration.

\section{Results \\ $R X R$ heterodimers containing $R A R, T R$, or $V D R$ preferentially select direct repeat elements}

To characterize the preferred core recognition motif sequences and orientations for different RXR heterodimers, high-affinity-binding sites were selected from synthetic oligonucleotides containing randomized sequences, as initially described by Blackwell and Weintraub (1990). The general strategy used was to randomize one recognition motif while providing a consensus AGGTCA motif at either the upstream or downstream location. The results of experiments utilizing an oligonucleotide probe containing the AGGTCA half-site at the upstream position followed by 11 degenerate base pairs are illustrated in Figure 1. Because of the extensive degeneracy of this probe, both palindromic and direct repeat organizations of the second half-site could potentially be selected at spacing intervals from 0 to $5 \mathrm{bp}$.

RXR/TR heterodimers strongly selected a direct repeat of the sequence $(\mathrm{A} / \mathrm{G} / \mathrm{GGTCA}$ at a spacing of $4 \mathrm{bp}$ from the fixed half-site (Fig. 1A,B). In addition to the selection of the $6 \mathrm{bp}$ corresponding to core recognition motif, partial selection also occurred in the spacer region between half-sites. In particular, strong selection against $A$ and $T$ was observed at randomized positions 3 and 4, respectively. Heterodimers of the RXR and VDR strongly selected the sequence AGTTCA as a direct repeat spaced $3 \mathrm{bp}$ from the upstream half-site. In contrast to the results obtained for RXR/TR and RXR/VDR heterodimers, an unambiguous sequence could not be obtained for RXR/RAR heterodimers, even after four rounds of selection and amplification (Fig. 1A). The selected and amplified pool of oligonucleotides exhibited markedly increased affinities for RXR/RAR heterodimers, however, indicating that a high degree of sequence selection had taken place (data not shown). To address whether highly selected variations of the core-binding motif were obtained, but at more than one spacing and/or orientation with respect to the first half-site, the PCR products obtained after one round of selection were subcloned and 16 individual clones were sequenced (Fig. 1C). Nearly all of the clones contained a selected motif that was related to the consensus AGGTCA recognition sequence. However, several different organizations of this motif were observed. The most frequent organization represented a direct repeat spaced $5 \mathrm{bp}$ from the first half-site (8/16). However, a direct repeat spaced by 2 bp was also observed at relatively high frequency (4/16). In addition, two sequences represented palindromic arrangements of the core motif: One sequence represented a direct repeat spaced by $1 \mathrm{bp}$, and one sequence was ambiguous. A direct comparison of the ability of RXR/RAR heterodimers to bind to recognition sequences consisting of AGGTCA spaced by 2 or 5 bp revealed similar binding affinities (data not shown).

These results indicated that RXR heterodimers preferentially selected direct repeat organizations of core recognition motifs. The precise sequence of the selected motif at the downstream binding site varied depending 


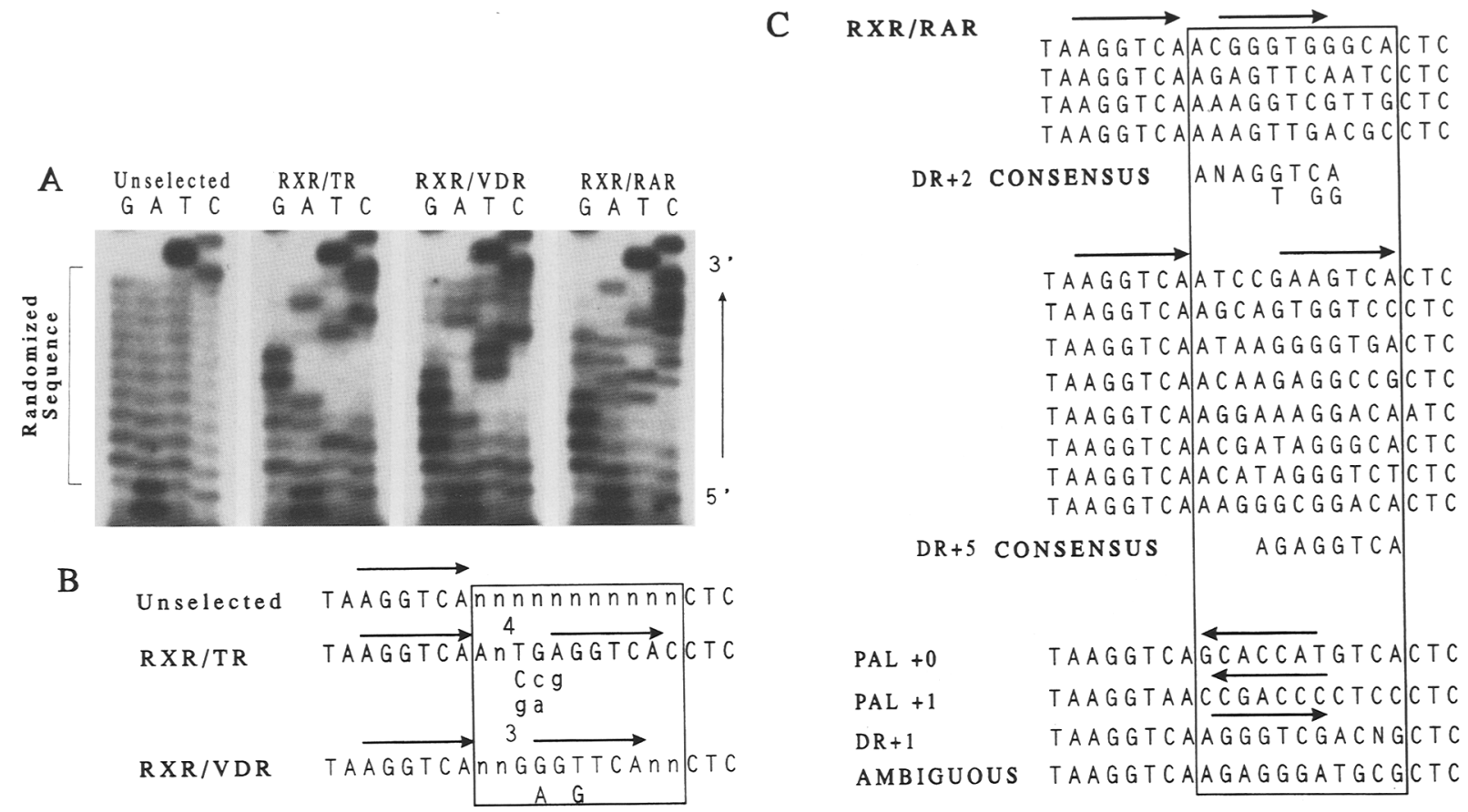

Figure 1. Selection of high-affinity-binding sites for RXR/RAR, RXR/TR, and RXR/VDR heterodimers. ( $A$ ) Selection of high-affinity sequences for RXR/VDR, RXR/TR, and RXR/RAR heterodimers at the downstream half-site. A double-stranded oligonucleotide containing a consensus AGGTCA recognition motif followed by 11 randomized base pairs was end-labeled with [ $\left.{ }^{32} \mathrm{P}\right] \mathrm{ATP}$ and incubated with RXR/RAR or RXR/TR heterodimers. Protein-DNA complexes were separated from free DNA by electrophoresis on nondenaturing polyacrylamide gels. Selected oligonucleotides were amplified using PCR, and the resulting amplified products were utilized for additional rounds of selection and amplification. The resulting pools of amplified oligonucleotides were sequenced directly using an antisense primer. The sequences illustrated for RXR/RAR were obtained after a total of four rounds of selection and amplification. The sequences for RXR/TR and RXR/VDR were obtained after two rounds of selection and amplification. $(B)$ The sequence of unselected SAAB 12 and summarizes the sequences obtained for RXR/TR and RXR/VDR heterodimers at the downstream half-site. The randomized and selected sequences are boxed. $(C)$ Sequences of individual binding sites selected by RXR/RAR heterodimers. Selected oligonucleotides obtained after one round of selection and amplification by RXR/RAR heterodimers were subcloned, and 16 independent isolates were selected for sequence analysis. These sequences are arranged according to the half-site spacing and orientation of a putative core-binding motif.

on the identity of the heterodimeric partner of RXRs. The sequence between the core recognition motifs also appeared to contribute to the specificity of binding of different heterodimers. Furthermore, the spacings between half-sites for each heterodimeric pair were consistent with the spacings between core recognition motifs that have been demonstrated previously to confer preferential regulation by retinoic acid, thyroid hormone, and vitamin D (Näär et al. 1991; Rottman et al. 1991; Umesono et al. 1991; Durand et al. 1992 and references therein).

To further define protein-DNA interactions between RXR heterodimers and direct repeat elements, methylation interference experiments were performed. Methylation interference patterns observed for RXR/TR heterodimers on a direct repeat element spaced by $4 \mathrm{bp}$ are illustrated in Figure 2A. N7 (major groove) methylation of guanidine residues within the downstream core recognition motif interfered with heterodimer binding to a greater extent than guanidine methylation within the upstream recognition motif. N3 (minor groove) methylation of adenosine residues within the spacer region between half-sites was also noted to interfere with RXR/
TR binding. The most significant degrees of interference were noted on the sense strand immediately $5^{\prime}$ of the core recognition motif. To examine minor groove contacts further, copper phenanthroline footprinting experiments were performed. These experiments demonstrated reduced cleavage immediately $5^{\prime}$ of each core recognition motif, consistent with minor groove interaction in these regions for both the RXR and TR (data not shown). Methylation interference analysis of RXR/RAR complexes bound to the $\beta$ RARE indicated a similar pattern of major and minor groove protein-DNA interactions (Fig. 2B). These experiments also demonstrated that minor groove methylation at the $5^{\prime}$ end of the upstream half-site interfered with RXR/RAR binding. In concert, these results suggest that minor groove contacts upstream of each of the two core recognition elements play an important role in DNA binding by RXR/RAR and RXR/TR heterodimers.

\section{Polarity and orientation of DNA-binding domains}

To determine whether specific receptors preferentially bound to the upstream or downstream half-site, a strat- 
Kurokawa et al.

A

$\mathrm{RXR} / \mathrm{TR}$ bound to $\mathrm{DR}+4$

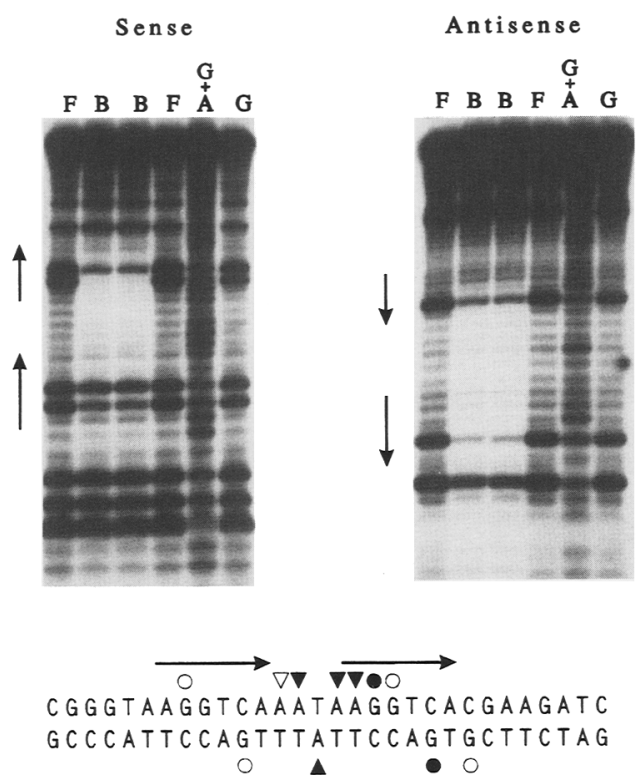

B

RXR/RAR bound to $\beta$ RARE

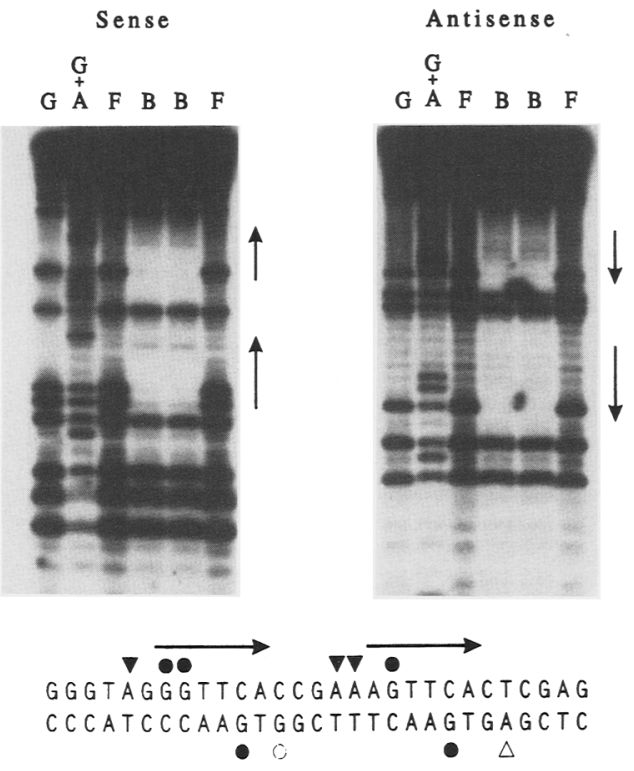

Figure 2. Identification of major and minor groove contacts of RXR/TR and RXR/RAR heterodimers. Oligonucleotides containing direct repeats of the AGGTCA core recognition motif spaced by 4 bp $(A, D R+4)$ or AGTTCA spaced by 5 bp $(B, \beta R A R E)$ were selectively labeled at either the sense (left) or antisense (right) strands and methylated with dimethylsulfate. The methylated oligonucleotides were incubated with RXR/TR $|A|$ or RXR/RAR heterodimers $(B)$, and protein-DNA complexes were separated on nondenaturing polyacrylamide gels. Bound $(B)$ and free $(F)$ DNA was recovered, cleaved with piperidine, and analyzed on sequencing gels. $\mathrm{G}$ and $\mathrm{G}+\mathrm{A}$ lanes were derived from conventional chemical sequencing reactions. Arrows denote the positions of the two recognition motifs. Circles represent positions of major groove interference caused by N7 methylation of guanine, and triangles represent positions of minor groove interference by N3 methylation of adenine. Solid symbols represent complete or nearly complete interference; open symbols represent weak interference (25-50\% of the intensity of corresponding band in free lanes).

egy was employed using radiolabeled oligonucleotides in which BrdU residues were introduced in or adjacent to one of the two half-sites. Protein-DNA complexes were separated from free DNA on nondenaturing polyacrylamide gels, irradiated with UV light, and analyzed for cross-linked products by SDS-polyacrylamide gel electrophoresis. Because the RXR, RAR, TR, and VDR are all of similar size, elongated versions of the RXR and RAR were constructed (termed RXR-Gt and RAR-Gt, respectively) to permit discrimination from their heteromeric partners on the basis of molecular mass. This was accomplished by fusing 200 amino acids containing the glucocorticoid receptor tau ${ }_{1}$ domain to the amino terminus of the RAR or RXR, as described previously /Glass et al. 1989). This addition of amino-terminal sequence did not alter the DNA-binding properties of either receptor (data not shown).

An experiment examining the cross-linking of RXR/ RAR-Gt heterodimers to BrdU-substituted retinoic acid response elements (RAREs) is illustrated in Figure 3A. Cross-linking of RAR-Gt was not BrdU-dependent and was therefore observed for both derivatized and underivatized RAREs. In contrast, cross-linking of RXR was only observed for substituted oligonucleotides containing BrdU in or near the first half-site. Cross-linking of RXR was not observed by oligonucleotides containing BrdU in or near the downstream half-site or by an underivatized RARE. Similar results were obtained for
RXR-Gt/TR heterodimers on derivatized and underivatized TR response elements (data not shown). These results provide direct evidence that the RXR preferentially binds to the upstream half-site as a component of RXR/ RAR or RXR/TR heterodimers.

On the basis of the preferential selection of direct repeat variants of the core AGGTCA recognition motif at the second half-site, it was likely that the DNA-binding domain of the receptor occupying this site was also oriented in a direct repeat configuration. However, studies of the structure of the glucocorticoid receptor DNAbinding domain complexed to a nonconsensus glucocorticoid response element (GRE) demonstrated that maintenance of protein-protein interactions at the dimerization interface took precedence over maintenance of sequence-specific protein-DNA interactions at one of the two half-sites (Luisi et al. 1991). It was therefore of importance to resolve whether the DNA-binding domain of the receptor occupying the downstream half-site made base pair interactions that were analogous to those of the receptor occupying the upstream half-site. To examine this question, an altered specificity mutant of the $T_{3}$ receptor was generated such that the P-box amino acids within the first finger of the $\mathrm{T}_{3}$ receptor DNA-binding domain (EGCKG) were replaced by the corresponding amino acids of the glucocorticoid receptor (GSCKV). The underlined valine residue is a critical determinant of GRE recognition because of van der Waals interactions 
A

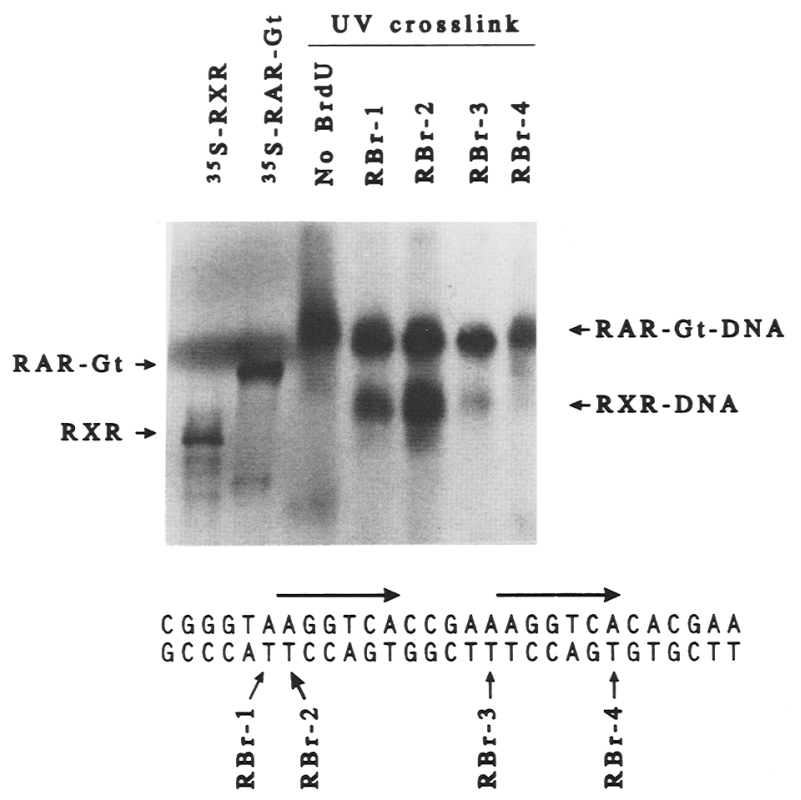

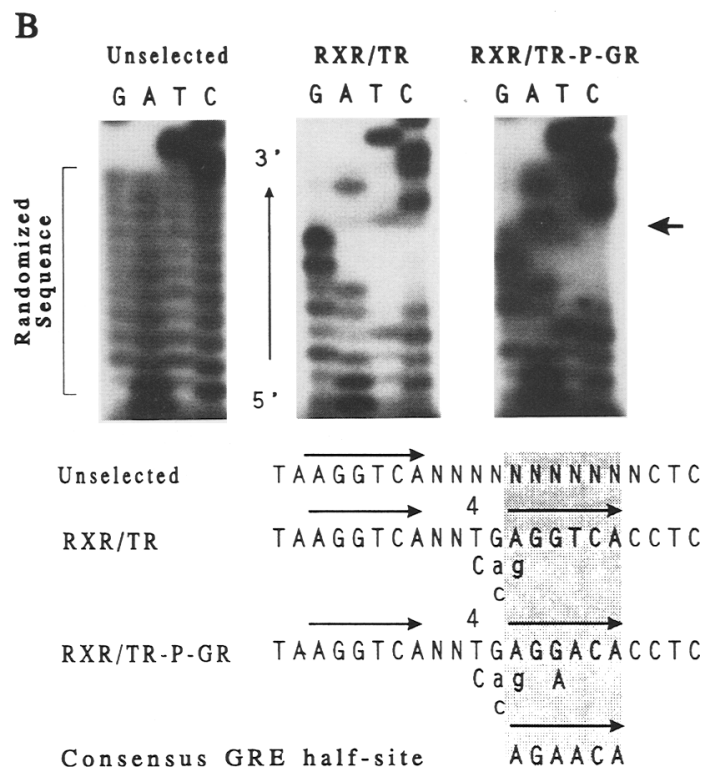

Figure 3. Polarity and orientation of RXR/RAR and RXR/TR heterodimer DBDs when bound to direct repeat elements. $(A)$ Preferential cross-linking of the RXR to the upstream half-site of a direct repeat of AGGTCA spaced by 5 bp. Double-stranded, synthetic oligonucleotides were prepared containing BrdU in place of thymidine at selected positions in either the upstream (RBr-1, RBr-2) or downstream (RBr-3, RBr-4) half-sites. These oligonucleotides were selectively labeled in the antisense strand and incubated with RXR/RAR-Gt heterodimers. Protein-DNA complexes were separated from unbound DNA by electrophoresis on nondenaturing polyacrylamide gels, irradiated with low wavelength UV light $(312 \mathrm{~nm})$ to affect protein-DNA cross-linking, and analyzed by electrophoresis on SDS-polyacrylamide gels. RAR-Gt is an elongated derivative of the RAR and was used in place of the wild-type RAR to permit the distinction of cross-linked products based on molecular mass. Lanes 1 and 2 illustrate the migration of in vitro-translated ${ }^{35} \mathrm{~S}$ labeled RXR and ${ }^{35}$ S-labeled RAR-Gt, respectively. $(B)$ Orientation of the $T_{3}$ receptor DBD over the downstream half-site of a DR +4 binding element. TR-P-GR is an altered specificity mutant of the TR in which the P-box residues of the TR were replaced with P-box residues of the glucocorticoid receptor. Wild-type and mutant TRs were incubated with SAAB 12 oligonucleotides in the presence of RXR, and selected oligonucleotides were isolated and amplified. The sequences obtained after two rounds of selection and amplification are illustrated. The arrow marks the base pair differences in sequences selected by the wild-type and mutant $T_{3}$ receptors.

between the valine side chain and the methyl group of the $\mathrm{T}$ residue corresponding to the $\mathrm{A} / \mathrm{T}$ base pair at position 4 of the AGAACA core recognition motif (Luisi et al. 1991). If the DNA-binding domain of the TR was aligned in a direct repeat configuration over the downstream half-site, the altered specificity mutant of the TR should select an $\mathrm{A}$ rather than a $\mathrm{T}$ at the fourth position of the core recognition motif.

The altered specificity mutant of the $\mathrm{T}_{3}$ receptor (TRP-GR) was capable of forming a heterodimeric complex with the RXR on the double-stranded oligonucleotide containing an AGGTCA half-site followed by 11 degenerate base pairs. Following two rounds of selection and amplification, the sequence $\mathrm{AG}\left({ }^{\mathrm{G}} / \mathrm{A}\right) \mathrm{ACA}$ was strongly selected as a direct repeat spaced $4 \mathrm{bp}$ from the upstream half-site (Fig. 3B). This selected sequence is indistinguishable from the consensus GRE core recognition motif and provides independent evidence that the DNAbinding domain of the TR is oriented as a direct repeat over the downstream half-site.

Dimerization properties of the $R A R, T R$, and $R X R$ independent of DNA

The previous results indicated that RXR heterodimers containing the RAR, TR, and VDR preferentially recognize direct repeat elements but did not provide insights into mechanisms by which binding was facilitated by the formation of heterodimers. One possible explanation for these observations was that heterodimers formed preferentially over homodimers. To address this possibility, a quantitative analysis of the dimerization properties of the RXR, RAR, and TR was performed (Fig. 4A). GST-RAR, GST-RXR, or GST-TR fusion proteins were adsorbed to microtiter wells and incubated with radiolabeled RAR, RXR, or TR produced by translation in vitro, and the quantities of specifically bound receptors were determined. In vitro-translated TR and RAR formed heterodimers with GST-RXR more readily than they formed homodimeric interactions (Fig. 4A). Conversely, the in vitro-translated RXR formed heterodimers more readily with GST-RAR and GST-TR than with GSTRXR. The relatively low affinities observed for RAR and RXR homodimers appear to result from a restrictive function imposed by their respective DNA-binding domains. The isolated carboxy-terminal ligand-binding/ dimerization domain of the RAR readily interacted with the GST-RAR fusion protein (Fig. 4A) and could be shown to form a homodimer in solution (Glass et al. 1990; data not shown), indicating that the DNA-binding 
A

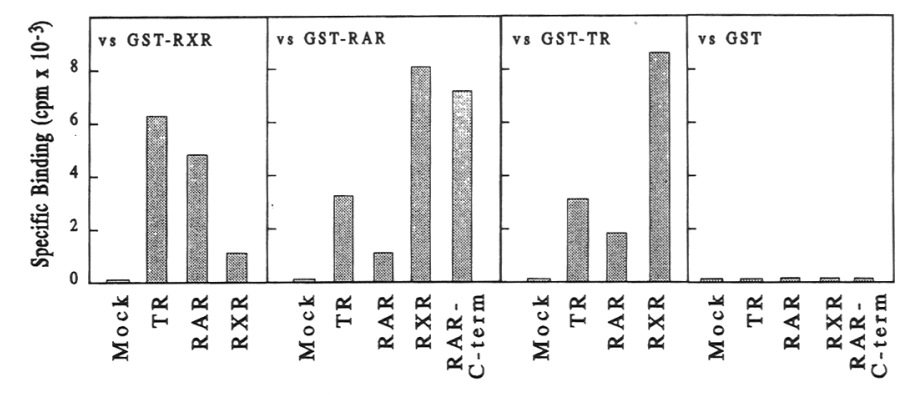

B

Figure 4. Preferential binding of the TR to direct repeat elements arises as a consequence of heterodimerization with the RXR. (A) Quantitative analysis of the strength of homodimerization vs. heterodimerization between TRs, RARs, and RXRs. TRs, RARs, and RXRs were produced as GST fusion proteins in E. coli and purified on a glutathione affinity matrix. The resultant fusion proteins, or GST alone, were adsorbed to microtiter wells. The wells were subsequently blocked with $5 \%$ serum and incubated with $100,000 \mathrm{cpm}$ of $\left[{ }^{35} \mathrm{~S}\right]$ methionine-labeled proteins produced by translation in vitro. The wells were washed rapidly four times, and remaining radioactivity was quantitated. Values represent the mean of duplicate points from a single experiment and are representative of the results obtained from three or more separate experiments. RAR carboxyl terminus represents the isolated carboxy-terminal dimerization interface. $(B)$ Binding properties of dimers and RXR/TR heterodimers to oligonucleotide probes containing different organizations of the core-binding motif. Left-facing arrows represent the core recognition sequence $5^{\prime}$-AGGTCA-3'. Right-facing arrows represent the inverse complement. Numbers between arrows represent the number of base pairs separating the core region recognition motifs. (See Materials and methods for complete sequences.) Equivalent amounts of TR and/or RXR protein $(\sim 20$ fmoles) were used in each $20-\mu \mathrm{l}$ binding reaction. Oligonucleotide probes were labeled to approximately equivalent specific activities and 200,000 cpm was utilized in each binding reaction. $(C)$ DNA-binding properties of the isolated TR-binding domain (codons 99-226). One and three microliters of purified protein were used in each $10-\mu l$ binding reaction, corresponding to $\sim 1$ and 3 pmoles of protein, respectively.

domain serves a role in restricting the actions of the carboxy-terminal dimerization interface. The observation that full-length receptors preferentially formed heterodimers over homodimers is therefore likely to account, at least in part, for the preferential binding of heterodimers to DNA.

\section{Preferential recognition of direct repeat elements by the $T R$ requires heterodimerization with the $R X R$}

Although the preceding experiments demonstrated that the TR preferentially interacted with the RXR, previous studies have demonstrated that the TR can bind as a homodimer to palindromic, direct repeat, and inverted

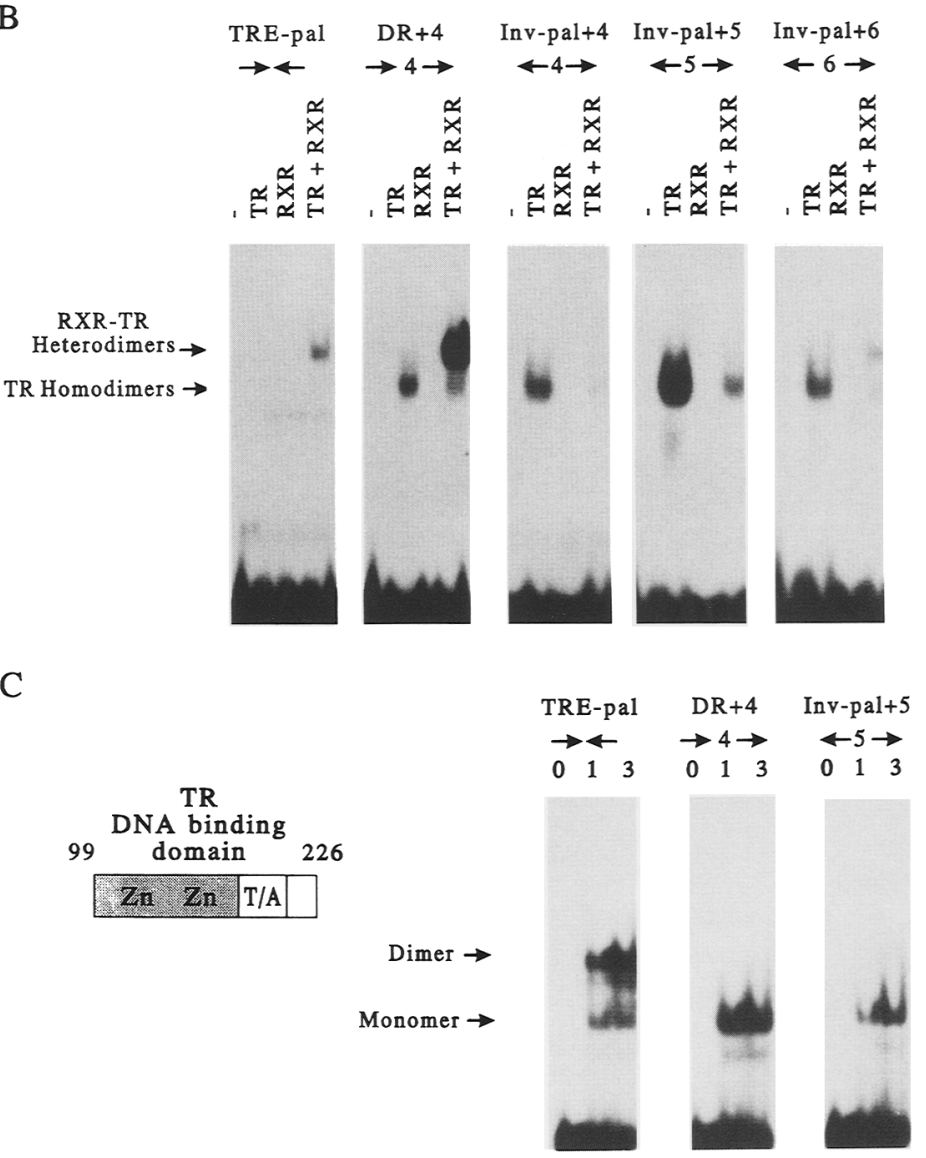

palindromic organizations of the core-binding motif (Baniahmad et al. 1990; Lazar et al. 1991; Näär et al. 1991). On the basis of these observations, we directly compared the ability of TR homodimers and RXR/TR heterodimers to bind to a series of response elements containing core recognition motifs arranged as direct repeats, palindromes, or inverted palindromes (Fig. 4B). Consistent with the results of the binding site selection assay, RXR/TR heterodimers preferentially bound to oligonucleotides containing direct repeat organizations of the core-binding motif spaced by $4 \mathrm{bp}$. RXR/TR heterodimers also bound to the palindromic arrangement (TRE-pal), although with a lower affinity than to the direct repeat elements. In contrast, oligonucleotides con- 
taining inverted palindromic arrangements of the core recognition motif at spacings of 4,5 , or 6 bp were recognized with high affinity by homodimers of the TR (Fig. 4B). Addition of RXR significantly reduced the binding of TR homodimers to the inverted palindrome sites, indicating that heterodimers formed in solution but had a lower affinity for the inverted palindrome arrangement than TR homodimers.

To confirm that inverted palindromes represented the preferred organizations of core recognition motifs for TR homodimers, binding site selection assays were performed using an oligonucleotide in which the downstream binding site was fixed and the upstream binding site was replaced by 12 randomized base pairs. After two rounds of selection and amplification, TR homodimers preferentially selected inverted palindromes of the core recognition motifs at spacings from 4 to $7 \mathrm{bp}$ from the downstream site (data not shown). These observations indicate that the preferential binding of the TR to direct repeat elements arose as a consequence of heterodimer formation with the RXR.

We then examined whether the preferential binding of the TR to inverted palindromes reflected an intrinsic property of its DNA-binding domain. To perform these experiments, the DNA-binding domain (DBD) of the TR was expressed in Escherichia coli and purified to near homogeneity. As expected, the TR DBD exhibited binding affinities for all organizations of core recognition motifs that were two to three orders of magnitude lower than those observed for the full length receptor, resulting from deletion of the carboxy-terminal dimerization function. Furthermore, the isolated DBD of the TR did not exhibit a preference for the inverted palindrome site, in marked contrast to the full-length TR (Fig. 4C). Even at micromolar concentrations, the TR DBD was observed to bind with similar affinities as a monomer to both the inverted palindrome and direct repeat-binding sites. Intriguingly, the TR DBD bound to TRE-pal as both a monomer and dimer, suggesting the presence of a symmetric dimerization interface mediating cooperative interactions on this element that was not available on direct repeat or inverted palindromic elements. These results indicate that the preferential binding of full-length TR homodimers to inverted palindromes is dependent on the carboxy-terminal dimerization interface and does not appear to result from cooperative interactions between the DBDs.

The differences in the DNA-binding properties of fulllength receptors and the isolated DBD suggested a simple model to account for the preferential binding of RXR/ TR heterodimers to direct repeat elements and TR homodimers to inverted palindromes of the core recognition motif. In this model, the DBD of the TR is predicted to be preferentially rotated $\sim 180^{\circ}$ with respect to the carboxy-terminal dimerization interface as compared with that of the RXR (see Fig. 8, below). As a result, the interactions between the carboxy-terminal dimerization interfaces of the TR and RXR would lead to the preferential alignment of their respective DBDs in a direct repeat arrangement and would serve to position RXR over the upstream binding site. In contrast, homodimeric interactions between the carboxy-terminal dimerization interfaces of the TR would lead to preferential alignment of the two DBDs in opposing directions, facilitating binding of homodimers to inverted palindrome organizations of the core recognition motif.

\section{A common dimerization interface is utilized for cooperative binding on palindromic, direct repeat, and inverted palindromic recognition elements}

The preceding model predicts that the identical TR carboxy-terminal dimerization interface is used for cooperative solution interactions that dictate binding to DNA elemer: ${ }^{+} \mathrm{c}$ containing direct repeat or inverted palindromic arrangement of core motifs. This model also raises a question as to whether an equivalent interface is used by the TR to interact cooperatively with the RXR on the palindromic (TRE-pal) DNA element. If so, it would require that the DBD of the TR be capable of rotating and bending with respect to the carboxy-terminal dimerization interface to permit cooperative interactions with the RXR carboxyl terminus. Alternatively, a second, independent dimerization interface could be used to interact with RXR on a site containing a palindromic arrangement of core DNA-binding motifs.

To test whether the same dimerization interface was used on direct repeat and inverted palindromes, the DNA-binding properties of TR mutants containing deletions, amino acid substitutions, or insertions within the carboxy-terminal dimerization/ligand-binding domain were tested for their ability to interact with the RXR on both palindromic and direct repeat elements. The effects of mutations in two regions of the carboxyl terminus of the TR that have been demonstrated previously to play important roles in dimerization are illustrated in Figure 5. Deletion of a short helical region within the extreme carboxyl terminus of the TR shown previously to be important in mediating interactions between TR/RAR heterodimers (TR $\Delta 422$; Glass et al. 1989) abolished its ability to form heterodimers with RXR on the TRE-pal and direct repeat elements, as well as homodimers on the inverted palindromes. Deletion of an internal region that is highly conserved among all members of the nuclear receptor superfamily and has been demonstrated to be critical for heterodimerization (O'Donnell et al. 1991) also abolished binding to all three classes of response elements. Further studies of a large panel of TRs containing various mutations within the carboxyl terminus were also most consistent with a model in which cooperative interactions on direct repeats, palindromes, and inverted palindromes depended on a single, large dimerization interface that is conserved across the nuclear receptor superfamily (data not shown) and that is capable of rotation with respect to the DBD.

As an independent strategy to determine whether single or multiple dimerization interfaces were used, oligonucleotides were synthesized containing three copies of the core recognition motif at various spacings and orientations with respect to one another. A receptor occupy- 
Figure 5. The TR involves the use of a single dimerization interface to interact cooperatively on palindromic, direct repeat, and inverted palindromic binding sites. The DNA-binding properties of TRs containing mutations in the carboxy-terminal dimerization domain were determined on direct repeat (MHC), palindromic (TRE-pal), and inverted palindromic (Inv-pal +4) binding sites. Binding conditions were identical to those described in Fig. 4B.

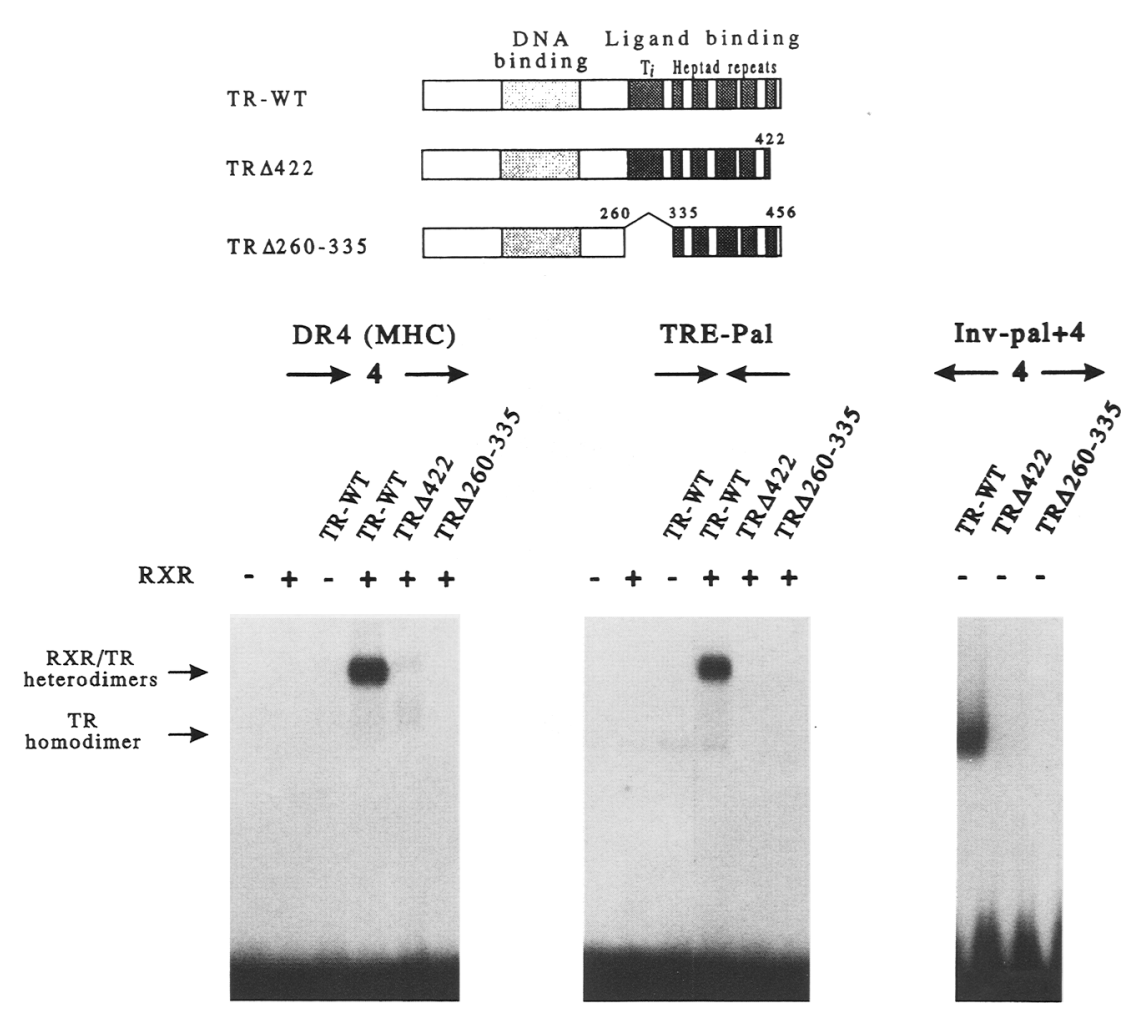

ing the central core recognition motif would therefore have the potential of simultaneously interacting with receptors bound to each of the flanking motifs if it contained multiple, independent dimerization interfaces. In no case, however, were cooperative interactions observed between three receptors on these binding sites (data not shown). Although these negative results do not provide conclusive evidence against the existence of multiple dimerization interfaces, they are consistent with the results of mutational studies (Fig. 5; Glass et al. 1989; Fawell et al. 1990) and the competition observed between receptor dimers and heterodimers on inverted palindromes (Fig. 4B), indicating that a single or overlapping dimerization surface is used for both homodimer and heterodimer formation, independent of the organization of the DNA recognition motif.

\section{Determinants of $D B D$ orientation}

To define amino acid sequences responsible for the differential orientation of the DBD with respect to the carboxy-terminal dimerization interface, a series of aminoand carboxy-terminal RXR-TR chimeras was generated. Analysis of these chimeras revealed that the orientation of the DBD was dependent on the identity of amino acids between the zinc finger and ligand-binding domains (data not shown), which encompass the $\mathrm{T}$ and $\mathrm{A}$ boxes (Wilson et al. 1992). This region was therefore examined in further detail in the cases of the RXR and TR. Transfer of 27 amino acids from the "hinge" region of the RXR (amino acids 198-224) into the corresponding region of the TR resulted in a chimeric TR (TR-H-RX) that retained the ability to interact with the RXR on the TREpal element but was unable to form homodimers on an inverted palindrome (Fig. 6B). Because the sequences of the core recognition motifs are identical for these two elements, we interpret this change in the relative binding preferences of TR-H-RX to reflect an altered preference in the orientation of the DBD with respect to the carboxy-terminal dimerization interface. Transfer of 9 amino acids containing the A-box region of the RXR (amino acids 213-220) into the TR resulted in a chimera (TR-A-RX) that exhibited decreased binding affinities for all three orientations of core recognition elements, with complete loss of binding observed for the inverted palindromic element (Fig. 6B).

These observations suggested that the amino acid region corresponding to the A box of the TR was also directly involved in protein-DNA interactions. Consistent with this possibility, nonconservative substitutions of 1 or 2 amino acids within this region dramatically reduced or abolished binding of TR homodimers to direct repeat or inverted palindromic elements (Fig. 7A). The same mutations reduced binding of RXR/TR heterodimers to both direct repeat and TRE-pal elements but to a much lesser extent on the direct repeat elements. The diminished effects of these mutations in the context of RXR/ TR heterodimer binding may reflect their presence in only one member of a heterodimeric pair.

Additional evidence that amino acids within or near the A box are critically involved in determining the preferred orientation of the DBD with respect to the car- 


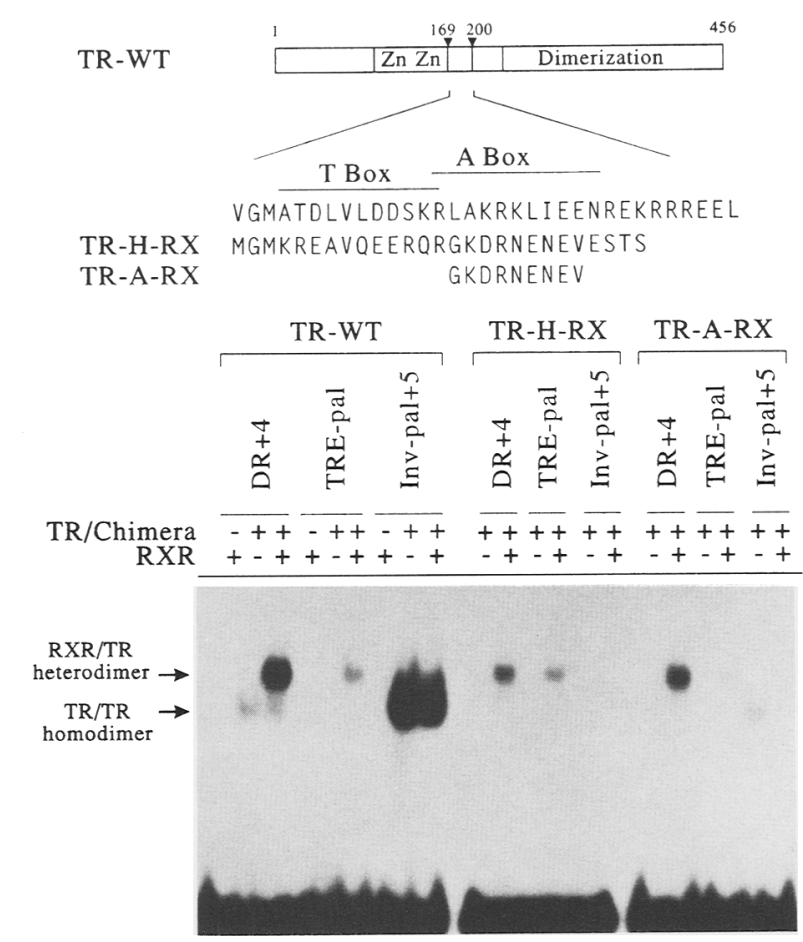

Figure 6. Role of the A-box regions in determining the DNAbinding preferences of the TR. DNA-binding properties of wildtype and chimeric TRs containing the hinge (TR-H-RX) or A-box (TR-A-RX) regions of the RXR on response elements consisting of direct repeat $(D R+4)$, palindromic (TRE-pal), and inverted palindromic organizations (Inv-pal +5 ) of the core recognition motif.

A
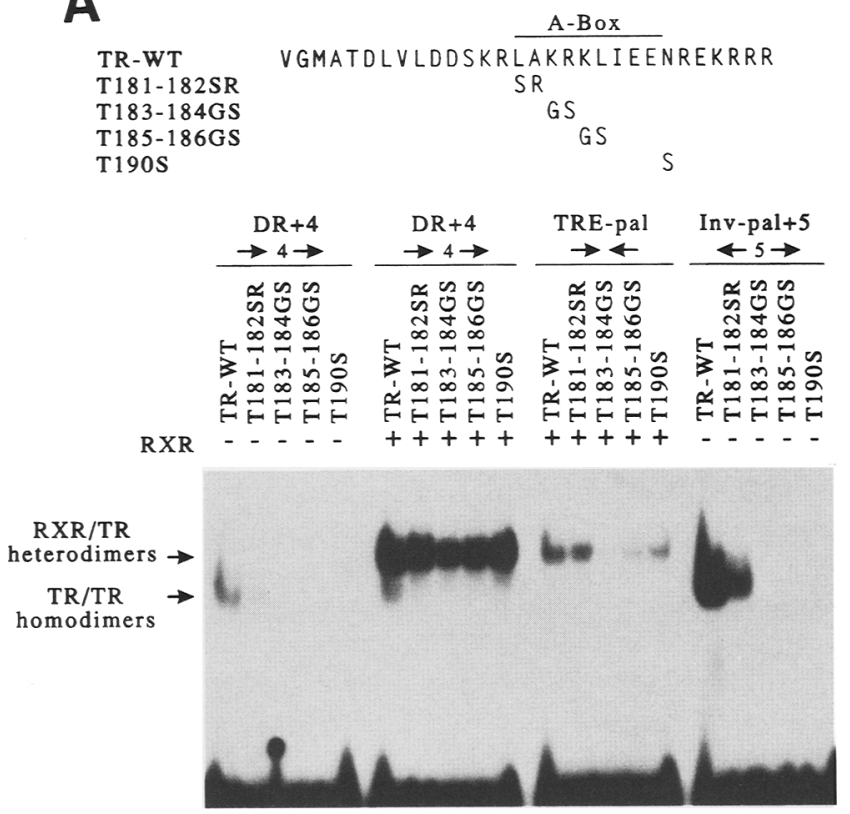

boxy-terminal dimerization interface was provided by the analysis of RXR mutants. Transfer of 9 amino acids containing the A box of the TR into the RXR resulted in a chimeric protein (RX-A-TR) that was capable of interacting more strongly with the TR on inverted palindromes (Fig. $7 \mathrm{~B}$; data not shown) than the wild-type RXR. Remarkably, introduction of a 2-amino-acid substitution at the carboxy-terminal end of the A box also resulted in a dramatic increase in the ability of the resulting chimera (RX219-220LE) to interact with the TR on inverted palindromic sites (Fig. 7B). Neither of these mutations reduced the ability of the RXR to interact with the TR on direct repeat or palindromic sites. However, the RXR mutant containing the TR A box (RX-ATR) could be cross-linked to both the upstream and downstream sites of direct repeat elements (data not shown). The loss of polarity on direct repeat elements observed for this RXR mutant is consistent with its ability to interact cooperatively with the TR on inverted palindromes. We therefore interpret this gain of function as being the result of disruption of a structure that maintains the preferred orientation of the RXR DBD with respect to the carboxy-terminal dimerization interface, allowing greater rotational flexibility.

\section{Discussion}

Preferential recognition of direct repeat elements by $R X R$ heterodimers

Previous studies have suggested that the sequence, spacing, and relative orientation of core recognition motifs

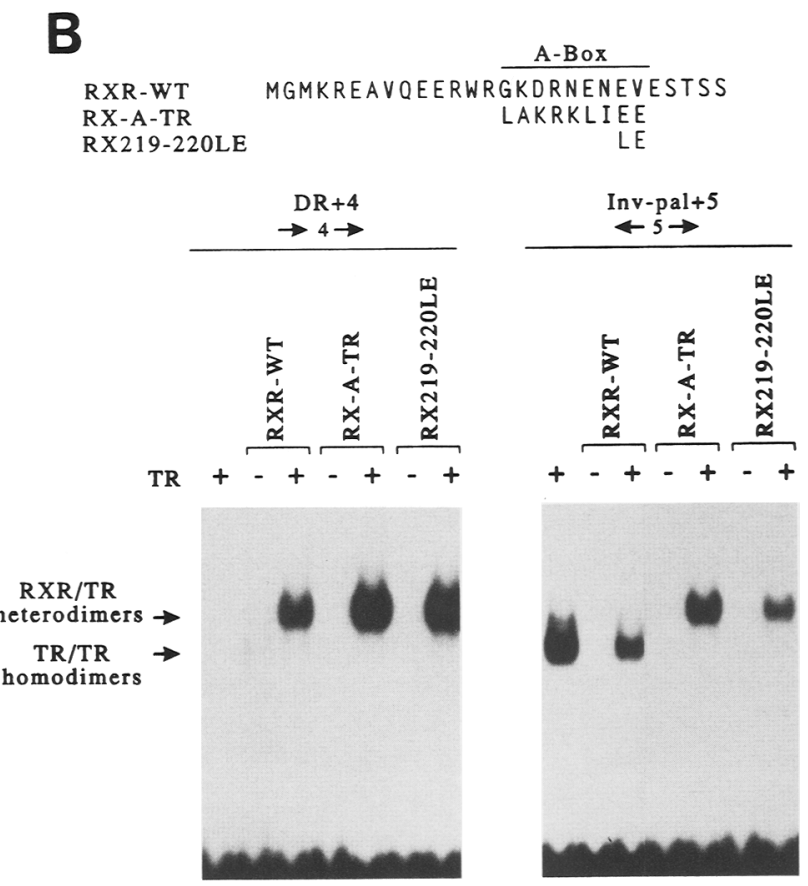

Figure 7. Role of the A-box region in determining high-affinity-binding and receptor polarity. $A$ illustrates the DNA-binding properties of TRs containing nonconservative mutations in the A-box region on direct repeat, palindromic, and inverted palindromic binding sites in the presence and absence of the RXR. B illustrates the DNA-binding properties of a RXR chimera containing the A box of the TR and a mutant receptor containing a 2 -amino-acid substitution. 
together determine the affinity and specificity of interactions between RARs, TRs, and VDRs and their respective response element sequences in target genes (Glass et al. 1988; Baniahmad et al. 1990; Lazar et al. 1991; Näär et al. 1991; Umesono et al. 1991; Forman et al. 1992; Carlberg et al. 1993; Tini et al. 1993). The studies presented in this paper indicate that the association of the TR, VDR, and RAR with RXR results in their preferential recognition of direct repeat elements. These DNAbinding properties appear to arise, at least in part, from the differential orientation of DBDs and the dimerization preferences of RXR, RAR, TR, and VDR. A comparison of the strength of dimerization of RXR, TR, and RAR homodimers versus heterodimers indicated that RXR heterodimers form preferentially, even in the absence of DNA. The same properties appear to be exhibited by RXR/VDR heterodimers (Z. Han, unpubl.). Thus, in a fashion analogous to fos and jun (Halazonetis et al. 1988; Rauscher et al. 1988; Kouzarides and Ziff 1989; Schuermann et al. 1989; Turner and Tjian 1989), heterodimeric binding reflects the relative dimerization preferences of each receptor.

Analysis of the dimerization properties of the TR are most consistent with a model in which both homo- and heterodimerization are mediated by a single complex interface that is utilized on both palindromic and direct repeat binding sites. These results imply that the DBD is capable of extensive rotation and bending with respect to the carboxy-terminal dimerization interface to allow cooperative interactions to occur on palindromic, direct repeat, and inverted palindromic binding sites. The present studies suggest that certain orientations are strongly preferred for each receptor. We propose that direct repeat elements represent the highest affinity-binding sites for RXR heterodimers because the DBDs of the RAR, TR, and VDR are preferentially rotated by $\sim 180^{\circ}$ with respect to the carboxy-terminal dimerization interface (Fig. 8A). These differences in the relative orientations of the DBDs with respect to the carboxy-terminal dimerization interface would lead to preferential alignment of the DBD in a direct repeat configuration, with RXR positioned to occupy the upstream core recognition motif (Fig. 8B).

\section{Role of the A box in sequence recognition and orientation of the $D B D$}

Our data suggest that the preferred orientations of the DBD are critically dependent on amino acid sequences located carboxy-terminal to the conserved zinc finger structures and overlap with sequences corresponding to the $\mathrm{T}$ and $\mathrm{A}$ box of RXR and NGFIb, respectively. As originally defined in the context of NGFIb, the A box consisted of amino acids that were required for selection of adenine residues at the $5^{\prime}$ end of the core recognition motif and that were necessary for high-affinity-binding of NGFIb monomers (Wilson et al. 1992). Point mutational analysis of the A-box region in the context of the TR demonstrated that it played a critical role in mediating high-affinity-binding of homodimers. The partial se-
A

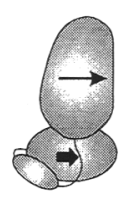

R X R

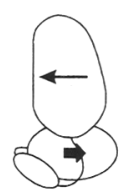

VDR

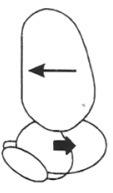

T R

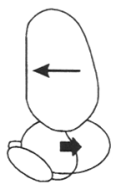

R A R

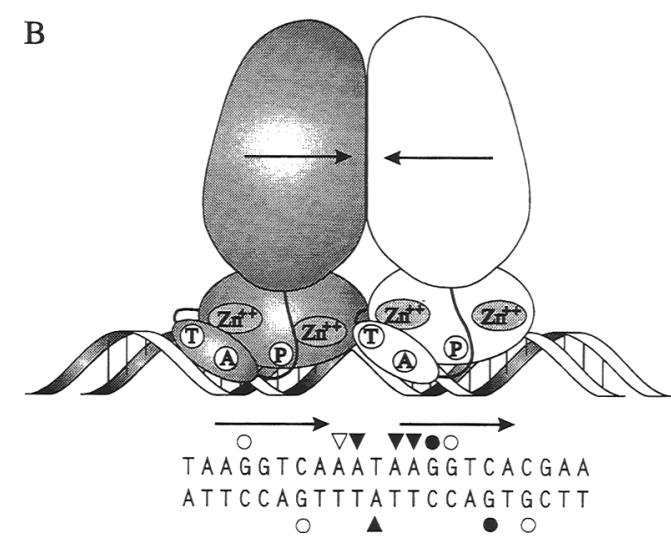

Figure 8. Model for RXR heterodimers bound to direct repeat and palindromic elements. $(A)$ The DBDs of the VDR, TR, and RAR are proposed to be rotated by $\sim 180^{\circ} \mathrm{C}$ with respect to the carboxy-terminal dimerization interface as compared with the RXR. Solution interactions between RXR and its heterodimeric partners mediated by the carboxy-terminal dimerization interface therefore lead to the alignment of their respective DBDs in a direct repeat configuration. (B) Model for an RXR/TR heterodimer bound to a direct repeat spaced by $4 \mathrm{bp}$. The RXR and TR are depicted as binding to the upstream and downstream recognition motifs, respectively. P-box residues with each receptor's $\mathrm{DBD}$ are predicted to make analogous contacts with the T/A base pairs at position 4 of the conserved recognition motif. A-box amino acids make minor groove contacts at the $5^{\prime}$ end of each core recognition motif and play a critical role in determining the relative orientations of the carboxy-terminal dimerization interfaces of RXR, RAR, TR, and VDR, perhaps directly contacting amino acids within the carboxyl terminus (not shown).

lection of sequences observed between half-sites and the minor groove contact analysis of RXR/RAR and RXR/ TR heterodimers bound to direct repeat elements suggest that the basic amino acids within the A box form protein-DNA contacts involving the minor groove and perform an important role in stabilizing DNA binding (Fig. 8B). This structural arrangement would be analogous to the utilization of minor groove interactions by homeo domain proteins (Kissinger et al. 1990). Mutations in the region corresponding to the $\mathrm{A}$ box of the RXR (amino acids 213-220) have not as yet demonstrated an equivalent role in mediating high-affinity DNA binding. Mutations in this region instead primarily increased the ability of RXR to interact with the TR on inverted palindromes and led to a loss of selective binding to the upstream half-site of direct repeat elements. These observations are consistent with the idea that this region of the RXR serves to orient the DBD with respect to the carboxy-terminal dimerization interface. 
Although the studies presented in this paper have focused on the role of the carboxy-terminal dimerization interface in mediating cooperative interactions that serve to discriminate among the relative orientations of core recognition motifs, several lines of evidence indicate that the DBD itself also contributes to the preferential binding of RXR heterodimers to direct repeat elements. First, the DBDs appear to contribute to the specificity of interactions that lead to preferential formation of heterodimers as compared with homodimers. Second, the observation that the spacings between recognition motifs are relatively restricted on direct repeats but not on inverted palindromes suggests the presence of a specific, asymmetric interface between heterodimeric DBDs on direct repeat elements that is distinct from the symmetric interface formed between steroid receptor DBDs. Recent studies of the isolated DBDs of the RXRs and RARs have demonstrated the presence of such an asymmetric interface that mediates cooperative interactions on direct repeat elements (T. Perlman and $\mathrm{R}$. Evans, pers. comm.). In concert, these observations suggest that dimerization driven by the carboxy-terminal interaction domain facilitates the formation of this asymmetric interface between the DBDs of RXR and its heterodimeric partner. These interactions, which are predicted to occur in solution, lead to the preferential binding of RXR heterodimers to response elements consisting of direct repeats of the core recognition motif.

We note that the differential-binding properties of homo- and heterodimeric receptors for response elements consisting of direct repeat, palindromic, and inverted palindromic arrangements of core recognition motifs are likely to have functional consequences. An inverted palindrome has recently been suggested to function as a response element for VDR receptor homodimers, whereas direct repeat elements are preferentially activated by RXR/VDR heterodimers /Carlberg et al. 1993). It will be of interest to determine whether the asymmetry of receptor occupancy on direct repeat elements will also have functional consequences with respect to the abilities of each receptor of a heterodimeric pair to interact with additional components of the transcriptional machinery.

\section{Materials and methods}

Expression of wild-type and mutant receptors

The receptor isoforms and amino acid numbering conventions used in these studies correspond to the following published sequences: RXR, human RXR $\alpha$ (Mangelsdorf et al. 1990); RAR, human RAR $\alpha$ (Giguere et al. 1987); TR, human TR $\beta$ (Weinberger et al. 1986); and VDR, human VDR (Baker et al. 1988). Except as noted, all nuclear receptors used in these studies were prepared by in vitro transcription/translation of wild-type and mutant receptor cDNAs as described previously (Yu et al. 1991). Parallel translation reactions were performed either with $\left.{ }^{35} \mathrm{~S}\right]$ methionine or unlabeled methionine, and the quantity of trichloroacetic acid (TCA) precipitable radioactivity from $\left[{ }^{35} \mathrm{~S}\right]$ methionine-containing translation reactions was used to normalize the amounts of unlabeled receptors used in DNAbinding assays.
Oligonucleotide-primed mutagenesis was performed by the method of Kunkel (1985), through the use of the helper phage R408 to rescue single-stranded template from pBluescript IIbased plasmids in the E. coli host strain CJ236. Xhol sites were introduced at the end of the T/A region of the TR at codons 188 and 189 , at codons 172 and 173 of the RAR, and at codons 219 and 220 of the RXR. Oligonucleotide-primed mutagenesis was also used to transfer the A- and T-box sequences between the RXR and TR and to introduce nonconservative changes in the A box of the TR. The DBD of the TR from codons 99 to 226 was expressed as a glutathione $S$-transferase (GST) fusion protein in E. coli. The fusion protein was purified to near homogeneity by affinity chromatography on a glutathione affinity matrix, and the TR DBD was cleaved from GST while attached to the affinity matrix using thrombin.

\section{DNA-binding assays}

Electrophoretic mobility-shift assays were performed utilizing each receptor at a concentration of $\sim 1 \mathrm{nM}$, as described previously (Yu et al. 1991). The bacterially produced TR DBD was used in DNA-binding assays at a concentration of $\sim 1 \mu \mathrm{M}$. The sequences of the sense strands of oligonucleotides used in electrophoretic mobility shift assays were as follows: $\beta$ RARE, AAGGGGATCCGGGTAGGGTTCACCGAAAGTTCACTCGAGATCTTCCT; DR2, AAGGGGATCCCTTGATCAGGTCATCAGGTCACAGATCTTCCT; DR4, AAGGGGATCCGGGTAAGGTCAAATAAGGTCACGAAGATCTGTT; DR4(MHC), AACAGATCTCCTTGGCTCTGGAGGTGACAGGAGCACAGCGGATCCCTT; DR5, AAGGGGATCCGGGTAAGGTCACAATAAGGTCACGAAGATCTGTT; TRE-pal, AAGGGGATCCGGTAAGATCAGGTCATGACCTGAGGAGATCTTCCT; INV-pal + 4, AAGGGGATCCCTTTGATCTGACCTGATCAGGTCACAGATCTTCCT; INV-pal +5, AAGGGGATCCCTTTGATCTGACCTGACTCAGGTCACAGATCTTCCT; INVpal +6, AAGGGGATCCCTTTGATCTGACCTGACGTCAGGTCACAGATCTTCCT; and PAL-INV, CTTTGATCAGGTCATGACCTGATCAGGTCACAGATCT.

Selection and amplification of high-affinity-binding sites for RXR heterodimers containing the RAR, TR, or VDR were performed essentially as described by Blackwell et al. (1990). SAAB 12 consisted of the sequence GATGAATTCCTAAGGTCANNNNNNNNNNNCTCGAGCTCAGATCTCGT, and PCR amplifications involved the use of primers P6 CGATGAATTCCTAAG and P2, TACGAGATCTGAGCTC. SAAB 10 consisted of the sequence CGATGAATTCCTAAGGGTCACCAGNNNNNNCTCGAGCTCAGATCTCGT, and PCR amplifications were performed using primers P6 and P2. Methylation interference assays were performed as described by Glass et al. (1988).

Cross-linking experiments were performed with synthetic double-stranded oligonucleotides containing single BrdU residues that were incorporated during oligonucleotide synthesis. BrdU cyanoethyl phosphoramidite was obtained from Pharmacia. The terminal trityl groups were maintained following synthesis, and the resulting oligonucleotides were purified using Nen-Sorb columns (New England Nuclear). All manipulations of BrdU-containing oligonucleotides were performed in a darkened room. The sequence of the control sense oligonucleotide used for cross-linking analysis of RXR/RAR heterodimers was CGGGTAAGGTCACCGAAAGGTCACACGAA. The positions of $\mathrm{BrdU}$ residues for $\mathrm{RBr}-1, \mathrm{RBr}-2, \mathrm{RBr}-3$, and $\mathrm{RBr}-4$ are illustrated in Figure 3.

For cross-linking experiments, the strand containing the BrdU residue was kinased with [ $\left.{ }^{32} \mathrm{P}\right] \mathrm{ATP}$ and annealed to its complement, and the resulting double-stranded oligonucle- 
otides were purified by nondenaturing polyacrylamide gel electrophoresis. Preparative scale electrophoretic mobility-shift assays were performed using BrdU-substituted or control oligonucleotides to separate protein-DNA complexes containing RXR heterodimers from free probe and background bands. UV crosslinking of the entire gel was performed using a Fisher transilluminator (model FBTIV-614, $\lambda=312 \mathrm{nM}$ ) at maximum intensity for $1 \mathrm{~min}$. The positions of bands corresponding to protein-DNA complexes containing RXR heterodimers were determined by autoradiography. These regions of the gel were carefully excised and heated at $55^{\circ} \mathrm{C}$ for $1 \mathrm{hr}$ in $100 \mu \mathrm{l}$ of SDS sample buffer. Samples were then heated at $100^{\circ} \mathrm{C}$ for and additional $3 \mathrm{~min}$ and inserted into the stacking gel of a SDSpolyacrylamide gel. After electrophoresis, cross-linked products were visualized by autoradiography. Exposure times ranged from 3 to 5 days.

\section{Quantitative analysis of protein-protein interactions}

To quantitate the strength of interactions between different nuclear receptors independent of DNA-binding, a quantitative microtiter dimerization assay was developed. In this assay, a primary nuclear receptor was adsorbed to the surface of a microtiter well and served as a target for a secondary radiolabeled receptor. The primary target receptors were produced by expression in E. coli as GST fusion proteins. These proteins were then purified to near homogeneity by affinity chromatography on a glutathione matrix. Purified target proteins were diluted to 20 $\mu \mathrm{g} / \mathrm{ml}$ in phosphate buffered saline (PBS) and adsorbed to a Dynatech 96-well microtiter plate for $2-12 \mathrm{hr}$ at $4^{\circ} \mathrm{C}$. Plates were then washed four times to remove unbound target receptor and residual nonspecific-binding sites were blocked by incubation with $5 \%$ serum in PBS for $2 \mathrm{hr}$ at $4^{\circ} \mathrm{C}$. Plates were then washed three additional times in PBS. Radiolabeled receptors produced by translation in vitro as described above were quantitated by precipitation with TCA, and the specified amounts were incubated with the target receptors in a volume of $50 \mu \mathrm{l}$ for $1 \mathrm{hr}$ at $4^{\circ} \mathrm{C}$. Binding buffer consisted of $20 \mathrm{~mm}$ HEPES $(\mathrm{pH} 7.8\}$, $50 \mathrm{~mm} \mathrm{KCl}, 20 \%$ glycerol, $0.1 \% \mathrm{NP}-40$ and $1 \mathrm{mM} \beta$-mercaptoethanol. Following the binding reaction, unbound receptor was rapidly removed from bound receptor by washing four times in binding buffer at $4^{\circ} \mathrm{C}$. Counts that remained bound to the plate were then released by incubation with buffer $\mathrm{H}$ containing $0.1 \%$ SDS and $0.4 \mathrm{M} \mathrm{HCl}$ and radioassayed.

\section{Acknowledgments}

We thank T. Perlman, K. Umesono, and R. Evans for sharing data before publication. We thank Xiang-Dong Fu, Jeffrey Holloway, and James DiRenzo for critical review of the manuscript and Michael Weiss for helpful discussion. We thank Sarah Flynn for assistance with site-directed mutagenesis and Nicole Boucher for assistance with preparation of the manuscript. A cDNA clone encoding human RXR $\alpha$ was generously provided by $\mathrm{S}$. Karathanasis. These studies were supported by grants from the National Institutes of Health (C.K.G., M.G.R.), the Howard Hughes Medical Institute (M.G.R.), and the Lucille P. Markey Foundation (C.K.G.). C.K.G. is a Lucille P. Markey Scholar.

The publication costs of this article were defrayed in part by payment of page charges. This article must therefore be hereby marked "advertisement" in accordance with 18 USC section 1734 solely to indicate this fact.

\section{References}

Baker, A.R., D.P. McDonnell, M. Hughes, T.M. Crisp, D.J. Man- gelsdorf, M.R. Haussler, J.W. Pike, J. Shine, and B.W. O'Malley. 1988. Cloning and expression of full-length cDNA encoding human vitamin D receptor. Proc. Natl. Acad. Sci. 85: 3297-3298.

Baniahmad, A., C. Steiner, A.C. Kohne, and R. Renkawitz. 1990. Modular structure of a chicken lysozyme silencer: Involvement of an unusual thyroid hormone receptor binding site. Cell 61: 505-514.

Beato, M. 1989. Gene regulation by steroid hormones. Cell 56: 335-344.

Blackwell, T.K. and H. Weintraub. 1990. Differences and similarities in DNA-binding preferences of MyoD and E2A protein complexes revealed by binding site selection. Science 250: 1104-1110.

Burnside, J., D.S. Darling, and W.W. Chin. 1990. A nuclear factor that enhances binding of thyroid hormone receptors to thyroid hormone response elements. I. Biol. Chem. 265: 2500-2504.

Carlberg, C., I. Bendik, A. Wyss, E. Meier, L.J. Sturzenbecker, J.F. Grippo, and W. Hunziker. 1993. Two nuclear signalling pathways for vitamin D. Nature 361: 657-660.

Durand, B., M. Saunders, P. Leroy, M. Leid, and P. Chambon. 1992. All-trans and 9-cis retinoic acid induction of CRABPII transcription is mediated by RAR-RXR heterodimers bound to DR1 and DR2 repeated motifs. Cell 71: 73-85.

Evans, R.M. 1988. The steroid and thyroid hormone receptor superfamily. Science 240: 889-895.

Fawell, S.E., J.A. Lees, R. White, and M.G. Parker. 1990. Characterization and colocalization of steroid binding and dimerization activities in the mouse estrogen receptor. Cell 60: 953-962.

Forman, B.M., J. Casanova, B.M. Raaka, J. Ghysdael, and H.H. Samuels. 1992. Half-site spacing and orientation determines whether thyroid hormone and retinoic acid receptors and related factors bind to DNA response elements as monomers, homodimers or heterodimers. Mol. Endocrinol. 6: 429-442.

Giguere, V., E.S. Ong, P. Segui, and R.M. Evans. 1987. Identification of a receptor for the morphogen retinoic acid. Nature 330: 624-629.

Glass, C.K. and M.G. Rosenfeld. 1991. Regulation of gene transcription by thyroid hormones and retinoic acid. In Molecular aspects of cellular regulation-The hormonal control regulation of gene transcription (ed. G. Foukes and P. Cohen), pp. 299-327. Elsevier Science Publishers B.V., Amsterdam, The Netherlands.

Glass, C.K., J.M. Holloway, O.V. Devary, and M.G. Rosenfeld. 1988. The thyroid hormone receptor binds with opposite transcriptional effects to a common sequence motif in thyroid hormone and estrogen response elements. Cell 54: 313323.

Glass, C.K., S.M. Lipkin, O.V. Devary, and M.G. Rosenfeld. 1989. Positive and negative regulation of gene transcription by a retinoic acid-thyroid hormone receptor heterodimer. Cell 59: 697-708.

Glass, C.K., O.V. Devary, and M.G. Rosenfeld. 1990. Multiple cell type-specific proteins differentially regulate target sequence recognition by the $\alpha$ retinoic acid receptor. Cell 63: 729-738.

Halazonetis, T.D., K. Georgopoulos, M.E. Greenberg, and P. Leder. 1988. c-Jun dimerizes with itself and with c-Fos, forming complexes of different DNA binding affinities. Cell 55: 917924.

Kissinger, C.R., B. Liu, E. Martin-Blanco, T.B. Kornberg, and C.O. Pabo. 1990. Crystal structure of an engrailed homeodomain-DNA complex at $2.8 \AA$ resolution: A framework 
for understanding homeodomain-DNA interactions. Cell 63 579-590.

Kliewer, S.A., K. Umesono, D.J. Mangelsdorf, and R.M. Evans. 1992. Retinoid X receptor interacts with nuclear receptors in retinoic acid, thyroid hormone and vitamin D3 signalling. Nature 355: 446-449.

Kouzarides, T. and E. Ziff. 1989. Leucine zippers of fos, jun and GCN4 dictate dimerization specificity and thereby control DNA-binding. Nature 340: 568-571.

Kunkel, T.A. 1985. Rapid and efficient site-specific mutagenesis without phenotypic selection. Proc. Natl. Acad. Sci. 82: 488-492.

Lazar, M.A., T.J. Berrodin, and H.P. Harding. 1991. Differential DNA-binding by monomeric, homodimeric and potentially heterodimeric forms of the thyroid hormone receptor. Mol. Cell. Biol. 11: 5005-5015.

Leid, M., P. Kastner, R. Lyons, H. Nakshatri, M. Saunders, T. Zacharewski, J.Y. Chen, A. Staub, J.M. Garnier, S. Mader, and P. Chambon. 1992. Purification, cloning, and RXR identity of the HeLa cell factor with which RAR or TR heterodimerizes to bind target sequences efficiently. Cell 68: 377-395.

Liao, J., K. Ozono, T. Sone, D.P. McDonnell, and J.W. Pike. 1990. Vitamin D receptor interaction with specific DNA requires a nuclear protein and 1,25-dihydroxyvitamin D3. Proc. Nat1. Acad. Sci. 87: 9751-9788.

Luisi, B.F., W.X. Xu, Z. Otwinowski, L.P. Freedman, K.R. Yamamoto, and P.B. Sigler. 1991. Crystallographic analysis of the interaction of the glucocorticoid receptor with DNA [see comments]. Nature 352: 497-505.

Mangelsdorf, D.J., E.S. Ong, J.A. Dyck, and R.M. Evans. 1990. Nuclear receptor that identifies a novel retinoic acid response pathway. Nature 345: 224-229.

Mangelsdorf, D.J., K. Umesono, S.A. Kliewer, U. Borgmeyer, E.S. Ong, and R.M. Evans. 1991. A direct repeat in the cellular retinol-binding protein type II gene confers differential regulation by RXR and RAR. Cell 66: 555-561.

Marks, M.S., P.L. Hallenbeck, T. Nagata, J.H. Segars, E. Appella, V.M. Nikodem, and K. Ozato. 1992. H-2RIIBP (RXR beta) heterodimerization provides a mechanism for combinatorial diversity in the regulation of retinoic acid and thyroid hormone responsive genes. EMBO J. 11: 1419-1435.

Murray, M.B. and H.C. Towle. 1989. Identification of nuclear factors that enhance binding of the thyroid hormone receptor to a thyroid hormone response element. Mol. Endocrinol. 3: 1434-1442.

Näär, A.M., J.M. Boutin, S.M. Lipkin, V.C. Yu, J.M. Holloway, C.K. Glass, and M.G. Rosenfeld. 1991. The orientation and spacing of core DNA-binding motifs dictate selective transcriptional responses to three nuclear receptors. Cell 65: 1267-1279.

O'Donnell, A.L., E.D. Rosen, D.S. Darling, and R.J. Koenig. 1991. Thyroid hormone receptor mutations that interfere with transcriptional activation also interfere with receptor interaction with a nuclear protein. Mol. Endocrinol. 5: 94 99.

Rauscher, F.J., P.J. Voulalas, B.R. Franza Jr., and T. Curran. 1988. Fos and Jun bind cooperatively to the AP-1 site: Reconstitution in vitro. Genes \& Dev. 2: 1687-1699.

Rottman, J.N., R.L. Widom, B. Nadal-Ginard, V. Mahdavi, and S.K. Karathanasis. 1991. A retinoic acid-responsive element in the apolipoprotein AI gene distinguishes between two different retinoic acid response pathways. Mol. Cell. Biol. 11: 3814-3820.

Schuermann, M., M. Neuberg, J.B. Hunter, T. Jenuwein, R.P. Ryseck, R. Bravo, and R. Muller. 1989. The leucine repeat motif in Fos protein mediates complex formation with Jun/ AP-1 and is required for transformation. Cell 56: 507-516.

Tini, M., G. Otulakowski, M.L. Breitman, L.-C. Tsui, and V. Giguere. 1993 An everted repeat mediates retinoic acid induction of the $\gamma \mathrm{F}$-crystallin gene: Evidence of a direct role for retinoids in lens development. Genes \& Dev. 7: 295-307.

Tsai, S.Y., J. Carlstedt-Duke, N.L. Weigel, K. Dahlman, J.A. Gustafsson, M.J. Tsai, and B.W. O'Malley. 1988. Molecular interactions of steroid hormone receptor with its enhancer element: Evidence for receptor dimer formation. Cell 55: 361-369.

Turner, R. and R. Tjian. 1989. Leucine repeats and an adjacent DNA binding domain mediate the formation of functional cFos-cJun heterodimers. Science 243: 1689-1694.

Umesono, K., V. Giguere, C.K. Glass, M.G. Rosenfeld, nd R.M. Evans. 1988. Retinoic acid and thyroid hormone induce gene expression through a common responsive element. Nature 336: 262-265.

Umesono, K., K.K. Murakami, C.C. Thompson, and R.M. Evans. 1991. Direct repeats as selective response elements for the thyroid hormone, retinoic acid, and vitamin D3 receptors. Cell 65: 1255-1266.

Weinberger, C., C. Thompson, E.S. Ong, R. Lebo, D.J. Gruol, and R.M. Evans. 1986. The c-erb-A gene encodes a thyroid hormone receptor. Nature 324: 641-646.

Wilson, T.E., R.E. Paulsen, K.A. Padgett, and J. Milbrandt. 1992. Participation of nonzinc finger residues in DNA-binding by two nuclear orphan receptors. Science 256: 107-110.

Yu, V.C., C. Delsert, B. Andersen, J.M. Holloway, O.V. Devary, A.M. Näär, S.Y. Kim, J.M. Boutin, C.K. Glass, and M.G. Rosenfeld. 1991. RXR beta: A coregulator that enhances binding of retinoic acid, thyroid hormone, and vitamin $D$ receptors to their cognate response elements. Cell 67: 12511266.

Zhang, X.-K., B. Hoffmann, P.B.-V. Tran, G. Graupner, and M. Pfahl. 1992. Retinoid X receptor is an auxiliary protein for thyroid hormone and retinoic acid receptors. Nature 355: 441-446. 


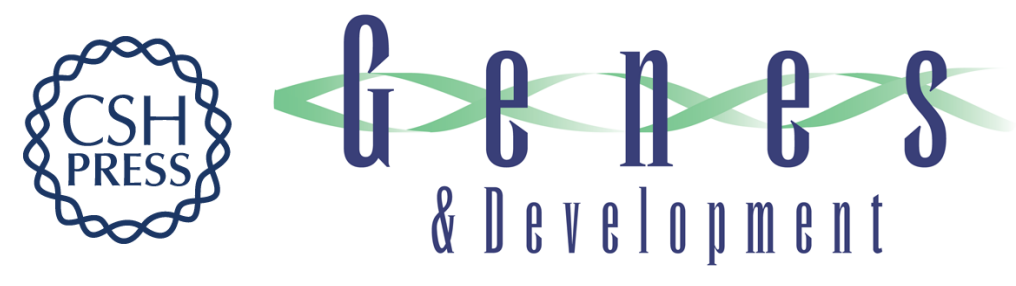

\section{Differential orientations of the DNA-binding domain and carboxy-terminal dimerization interface regulate binding site selection by nuclear receptor heterodimers.}

R Kurokawa, V C Yu, A Näär, et al.

Genes Dev. 1993, 7:

Access the most recent version at doi:10.1101/gad.7.7b.1423

References This article cites 41 articles, 11 of which can be accessed free at:

http://genesdev.cshlp.org/content/7/7b/1423.full.html\#ref-list-1

License

Email Alerting

Receive free email alerts when new articles cite this article - sign up in the box at the

Service top right corner of the article or click here.

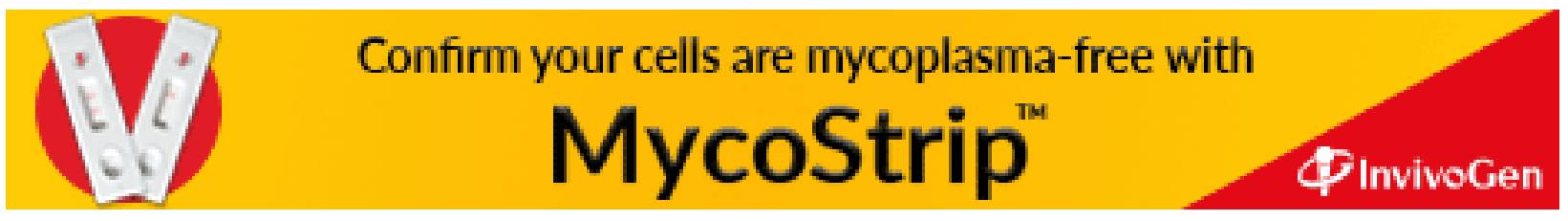

